\title{
O PROBLEMA DA AUTOADMINISTRAÇÃO DE MEDICAMENTOS POR IDOSOS COM BAIXA VISÃO E CEGUEIRA SOB A ÓTICA DO DESIGN CENTRADO NO HUMANO
}

Fernanda Jordani Barbosa Harada

Universidade Federal de São Paulo/UNIFESP

ferjordani@gmail.com

Paulo Schor

Universidade Federal de São Paulo/UNIFESP

paulo.schor@gmail.com

Resumo: Este artigo relata achados iniciais de uma investigação de caráter transdisciplinar entre duas áreas de competências distintas: medicina e design. $\mathrm{O}$ aumento do número de idosos é uma realidade global que abre precedentes para diversas frentes de pesquisa. Como método para pesquisa exploratória foram utilizados conversas informais guiadas por entrevistas semiestruturadas para compreender problemas relacionados à incorreta autoadministração de medicamentos por idosos com deficiência visual. Os achados apontam a falta de padrão nos medicamentos distribuídos na rede pública e sugerem pré-requisitos para desenvolvimento de soluções que auxiliem estes idosos, minimizem erros e promovam sua independência.

Palavras-chave: Baixa Visão; Cegueira; Design Inclusivo; Design Centrado no Humano; Idosos

\begin{abstract}
The increasing number of elderly is a global reality in which opens precedents for various research fronts. This article reports the initial findings of a transdisciplinary research that connects two distinct areas of expertise: medicine and design. The increasing number of elderly is a global reality in which opens precedents for various research fronts. In order to understand problems related to the incorrect self-administration of medication by elderly with visual impairment, it was used informal conversations conducted by semi-structured interviews as an exploratory research method. The findings pointed out the lack of standards in distributed medicines by the public system and suggest some prerequisites for developing solutions to support these elderly, minimizing misusage and promoting their independence.
\end{abstract}

Keywords: Low Vision; Blindness; Inclusive Design; Human Centered Design; Elderly 


\title{
1. INTRODUÇÃO
}

\subsection{Envelhecimento Populacional}

O envelhecimento populacional é um processo global e não regional, e implica em significativa mudança sócio demográfica do mundo atual e considerável aumento da expectativa de vida do homem ao longo dos anos. Este acelerado crescimento do número de idosos é uma realidade que tem profundas implicações para a nossa sociedade, e deve ser encarado como um importante desafio na busca de novas e criativas soluções que aumentem a qualidade de vida e autonomia destes idosos (Clarkson et al., 2003 ; Kalache, 2013; Fletcher, 2014b).

Segundo o Centro Internacional de Longevidade Brasil (ILC, 2015) o crescimento da população de idosos em números absolutos e relativos, é um fenômeno mundial que ocorre em um nível sem precedentes. Em seu sumário executivo no relatório de Síntese Global de 2015 o Índice da AgeWatch ressalta,

\begin{abstract}
Apesar de nem sempre o reconhecermos, o envelhecimento da população mundial é a maior história de sucesso de desenvolvimento humano, tendo resultado da queda das taxas de natalidade e da maior esperança de vida. Contudo, até agora nem todos os governos criaram um enquadramento político para responder aos desafios colocados pelo envelhecimento das suas populações. (Global Agewatch Index, 2015)
\end{abstract}

O perfil demográfico do Brasil segundo o IBGE, "ainda terá uma longa jornada de transformações" pois a redução da fecundidade em ação conjunta com a queda da mortalidade, fazem com que a esperança de vida ao nascer aumente e consequentemente produza uma elevação futura do número de pessoas que chegam à terceira idade. A expectativa de vida da população brasileira ao nascer evoluiu de 66,93 anos em 1991 para uma previsão de 78,33 anos em 2030 (IBGE, 2008). O envelhecer, portanto, passa de simples fato na vida de um ser humano, para uma realidade demográfica que grande parcela da população mundial, da sociedade e seus serviços não estão preparados para acolher (Clarkson et al., 2003 ; Fletcher, 2016).

Paralela a esta transição demográfica ocorre uma transição epidemiológica pois ao aumento da idade média do idoso correspondem à mudanças na incidência e prevalência de doenças (Kalache, 2013). Este processo causa o aumento gradual da coexistência de transtornos, estados patológicos ou doenças, que influenciam no declínio funcional e comprometimento da qualidade de vida das pessoas - as chamadas comorbidades (Zaitune et al., 2006). Dentre estas, a diabetes mellitus tipo 2 se apresenta entre as doenças mais comuns no universo do idoso e que se transforma em um grave problema de saúde pública (Mendonça et al., 2008).

Dados estatísticos da Organização Mundial de Saúde (OMS) mostram que o número de diabéticos no mundo passará de 171 milhões em 2000, para uma previsão de 366 milhões em 2030. Sendo que neste cenário o Brasil ocupa a sétima posição em número de diabéticos entre 35 e 64 anos, e no ano de 2030 chegará a 11,3 milhões (Belon, 2008). De acordo com o Centers for Disease Control and Prevention esta doença é apontada como uma das principais causas de cegueira entre adultos com idade de 20 a 74 anos. Vale salientar que a diabetes quando mal controlada, pode levar à doença vascular e suas complicações como infarto do miocárdio, acidente 
vascular cerebral, insuficiência arterial periférica e Retinopatia Diabética (RD) comprometimento dos vasos da retina e consequente perda visual (CDC, 1996).

Além da RD, uma das principais causas de perda de visão em pessoas com idade acima de 50 anos é a Degeneração Macular Relacionada a Idade (DMRI), complicação desencadeada por uma série de fatores genéticos, metabólicos e ambientais e que acomete a área central da retina, chamada mácula, evoluindo assim para a perda visual progressiva (Sampaio e Haddad, 2009). No Setor de Baixa Visão e Reabilitação Visual do Departamento de Oftalmologia e Ciências Visuais da Universidade Federal de São Paulo/Escola Paulista de Medicina, a DMRI representa a principal demanda de pacientes adultos para reabilitação visual.

Vale salientar que a grande incidência de doenças crônicas em idosos levam ao aumento do consumo de medicamentos (Jannuzzi, 2009), e estudos mostram que destes idosos, aqueles que apresentam algum tipo de problema visual têm duas vezes mais chance de precisar apoio para uso de medicações (Mccann et al., 2012). Estes medicamentos devem ser rigorosamente administrados, pois alterações farmacodinâmicas e farmacocinéticas de fármacos no organismo levam ao aumento de interações medicamentosas, reações, efeitos colaterais e complicações extremas (Loyola Filho et al., 2006). Logo, são necessárias reformas físico-ambientais, sociais, políticas e na saúde que foquem na melhoria das condições de vida desta população, otimizando atividades de vida diária destes idosos e amenizando as restrições e impedimentos sucedidos com a idade (Fletcher et al., 2015). Este acontecimento portanto, deve ser repensado em diversas frentes de pesquisa, e em uma abordagem centrada no ser humano, para não se tornar um grave problema de saúde pública.

\subsection{Design Centrado no Humano}

O desenvolvimento de um projeto para o designer, tem como meta o bemestar do usuário. Portanto uma solução de projeto universal, para ser bem sucedida, requer uma investigação criteriosa por parte do designer quanto aos aspectos comportamentais, cognitivos e sensoriais do grupo de usuários em questão (Fletcher et al., 2015). Para isso, a observação de experiências humanas e compreensão de suas demandas exige uma pesquisa detalhada e um amplo esforço de investigação do indivíduo pelo designer para daí criar soluções que o satisfaçam (Postma et al., 2012). Desta forma, Design Centrado no Humano (DCH) vai além do processo de design tradicional, e explora os fatores humanos por trás do desenvolvimento de um projeto a fim de entender a experiência das pessoas e como estas interagem com o meio e suas soluções (Giacomin, 2012). Esta abordagem auxilia o designer a alcançar o mais amplo espectro de pessoas através da concepção de projetos que atinjam a maior variedade de necessidades (Eikhaug et al., 2010).

Existe uma distinção amplamente debatida em projetos de design que tem o indivíduo como foco principal do projeto. O DCH e Design Centrado no Usuário (DCU) possuem referências teóricas muito semelhantes, pois se utilizam de técnicas e processos para se aproximar do indivíduo - que é ou será -usuário de determinada solução, e projetar soluções com boa usabilidade (Chaves, 2014). Segundo Norman (2002) o DCU é uma filosofia baseada nas necessidades dos usuário e seus interesses. Para (Fletcher et al., 2015), tanto DCH como DCU, trabalham com técnicas que ultrapassam a ideia básica de identificação de possíveis problemas ou melhoria de produtos e focam em colocar o indivíduo ou usuário no centro do processo, para 
pensar primeiro em seres humanos para então projetar soluções para seu dia a dia. Além disto, utilizam a interação do indivíduo para guiar suas necessidades e propor saídas sem que este tenha a consciência deste papel (Leonard e Rayport, 1997).

Sob o guarda-chuva do $\mathrm{DCH}$, há uma ampla gama de abordagens a fim de estabelecer uma conexão com futuros usuários e compreender melhor suas ações, necessidades e preferências. Uma das maneiras mais eficazes é através da observação e empatia para estabelecer conexão com o indivíduo (Suri, 2003). Em outras palavras, a abordagem de design empático tem sido utilizada como uma ferramenta para enriquecer a compreensão do indivíduo pelo $\mathrm{DCH}$ e $\mathrm{DCU}$, e consequentemente aumentar o poder criativo do designer (Postma et al., 2012). Para seguir este caminho, existem três principais abordagens: primeiro é olhar para o que as pessoas realmente fazem: realizar uma profunda observação do usuário in loco e no seu contexto, pois uma observação detalhada informa a imaginação subjetiva e estimula a criação; segundo é pedir às pessoas para participar: gravando seu comportamento, expressões, sentimentos e pensamentos sobre um conceito ou um protótipo; terceiro na tentativa de obter insights pessoais sobre experiências que outros já tiveram, experimentar diferentes situações individualmente (Suri, 2003).

Pesquisas em design portanto, buscam analisar e avaliar o universo do indivíduo para propor soluções que otimizem sua experiência com o ambiente. Neste sentido, o ambiente, assim como definido pela Organização Mundial de Saúde - OMS, de forma holística como tudo o que compõe o local onde o indivíduo está inserido, ou o meio em que cada um vive composto de: ambiente físico, informações, comunicações, serviços, atitudes e políticas (Fletcher, 2016). Neste contexto, o design universal passa a ser uma estratégia na concepção soluções inclusivas, pois o uso de abordagens empáticas e centradas no ser humano oferecem vantagens sobre métodos tradicionais para compreender, avaliar e criar soluções para problemas (Clarkson et al., 2003 ). Assim, torna-se fundamental posicionar o indivíduo (ou usuário) com um papel ativo e participativo no centro do processo de pesquisa dentro de seu ambiente,

A OMS definiu desenho universal como a estratégia mais promissora dos últimos tempos. Desenho universal, também chamado de design inclusivo, ou o design para todos, é uma forma de pensamento que coloca as pessoas no centro do processo de design. É a base para o design de lugares, coisas, informação, comunicação e políticas que incidem sobre o usuário, e sobre a mais ampla gama de pessoas que operam na mais ampla gama de situações, sem um design especial ou separado.(Fletcher, 2014a)

Neste processo, somente o uso de dados antropométricos e ergonômicos hoje são insuficientes para explicar as reais necessidades dos usuários (Fletcher, 2014b). 0 humanismo projetual é um meio de interpretar as necessidades de grupos sociais pelo designer e elaborar propostas viáveis e emancipatórias equilibrando aspectos técnicos e semânticos dos objetos para atender a necessidade do usuário, e consequente gerando bem estar pelo uso (Bonsiepe, 2011). Esses métodos mudam a mentalidade de projetar "para pessoas", para projetar "com pessoas", tendo o usuário como parceiro em todo o processo, antecipando necessidades e proporcionando uma aprendizagem enriquecida de experiências ao projeto e à equipe de pesquisa transdisciplinar (Postma et al., 2012). 
Segundo (Turato, 2008) "sentidos e significações dos fenômenos são o cerne para os pesquisadores qualitativistas (...) e procurar capturá-los, ouvindo e observando os sujeitos da pesquisa, bem como dar as interpretações, são os objetivos maiores". Da mesma forma que métodos quantitativos buscam a explicação das coisas e seu objeto de estudo são fatos naturais descritos, a metodologia qualitativa busca a compreensão do homem e tem nos fenômenos humanos apreendidos seu objeto de estudo. A valorização das angústias e ansiedades dos pacientes, é fundamental. Desta forma, afirma o autor:

Tratando-se do uso do método qualitativo aplicado em settings da saúde, é imprescindível ao investigador, acolhendo a pessoa numa atitude clinica, valorizar a existência das angustias e ansiedades da pessoa entrevistada como um elemento fundamental de mobilização do interesse do entrevistador. E são as próprias angústias e ansiedades do pesquisador que igualmente o movem para querer entender as leis das manifestações humanas. (Turato, 2008, p. 252).

Portanto, concepções básicas para o estudo do homem e da saúde na abordagem metodológica clínico qualitativa exigem do pesquisador um enfoque mais humano e portanto a identificação de questões sensíveis no contexto estudado. Quando o sujeito de pesquisa atua como um perito, neste tipo de abordagem qualitativa, este é intitulado de User/Expert (U/E), ou seja, aquele que explora o campo e dá pistas ao projeto de pesquisa baseadas em sua experiência. O U/E é portanto, extremamente valioso para estudos, principalmente quando este tem uma limitação funcional determinada, tais como descritos:

User/Experts são pessoas que através de suas experiências com o meio, desenvolveram naturalmente e espontaneamente conhecimento sobre como lidar com barreiras no seu dia a dia, como por exemplo, desafios presentes no ambiente construído. Neste contexto e em busca de gerar padrões universais, a experiência e conhecimentos únicos dos User/Experts são características essenciais para a especificação precisa de problemas e constante melhora na definição e solução de problemas (Froyen, 2012, p. 173).

Um projeto que tem o U/E como parceiro, utiliza investigações contextuais profundas sobre o "ser" e seu ambiente a ser investigado. Segundo Fletcher (2014):

Não importa o quão comprometido e experiente é o profissional designer, não há substituto para envolver usuários reais com a maior variedade de experiências pessoais e limitações funcionais, para interagir com ambientes e enriquecer os achados da pesquisa (Fletcher, 2014a, p.2).

A seguir, mostraremos as observações realizadas em pacientes do programa de saúde pública especializado da UNIFESP/EPM nos ambulatórios do Departamento de Oftalmologia. O objetivo principal foi compreender problemas relacionados à incorreta autoadministração de medicamentos na população idosa com baixa visão e cegueira, portadores de doenças crônico-degenerativas e usuários de múltiplos fármacos. 


\section{MÉTODOS}

Em busca de compreender as demandas, dinâmica diária e relações pessoais de idosos com problemas visuais e seus medicamentos, este projeto utilizou metodologia de pesquisa qualitativa com abordagem centrada no humano e conversas informais guiadas por questionário semiestruturado. Após as conversas, para avaliar e delinear o problema e obter conclusões metodologicamente mais precisas, foram utilizados dois tipos de ferramentas metodológicas: stakeholder mapping (mapeamento das partes envolvidas) e abstraction later (escada da abstração).

\subsection{Sobre Conversas Informais}

Conversas informais, são abordagens que fazem parte de métodos de observação e estratégias de campo muito comuns em pesquisa qualitativa. Devem ser conduzidas com naturalidade e as questões devem ser introduzidas de modo fácil sem ser intrusivo e previsível. O observador deve ser disciplinado e não interferir e inferir nos significados presentes na fala dos participantes, bem como não preestabelecer e fechar a conversa somente em questões a serem respondidas (Patton, 2001). Desta forma, as conversas informais constituem em uma importante ferramenta de trabalho de campo com usuários, por isso que muitas vezes é referido como "entrevista etnográfica". Esta abordagem gera dados que diferem de pessoa para pessoa, "uma abordagem que funciona particularmente bem quando o pesquisador pode estar em campo por um bom período de tempo, para não depender apenas de uma única oportunidade de entrevista. (Patton, 2001, p.342)

Segundo Patton (2001), este tipo de abordagem deve oferecer flexibilidade máxima para buscar informações em qualquer direção que se mostra mais apropriada, pois depende "do que emerge da observação de um ambiente particular ou da fala de um ou mais indivíduos nessa configuração", para assim as perguntas fluírem a partir do contexto imediato". Portanto, a forma como o pesquisador aborda o indivíduo interfere diretamente no próximo estágio da pesquisa,

Embora você esteja tentando compreender demandas para gerar ideias, não trate as pessoas como 'sujeitos de um teste'. Garanta que os usuários/especialistas sejam vistos como valiosos contribuintes e sejam respeitados. Os insights que recolhidos nesta fase serão uma plataforma para a inspiração dos próximos passos. (Eikhaug et al., 2010, p.46)

A pesquisa utilizou uma lista de questões a serem abordadas para conduzir as conversas, mas nunca limitando os assuntos e temas abordados pelos pacientes.

\subsection{Assuntos Abordados}

Foi utilizado uma lista assuntos para direcionar as conversas informais e abordar temas relativos ao indivíduo, seu tratamento no ambulatório e assuntos específicos ao seu dia a dia na administração de seus medicamentos (quadro 1). A primeira parte da conversa abordou assuntos práticos, como conhecimento do tempo de doença e outras doenças, percepção sobre sua condição visual e tratamentos que foram já realizados. A segunda parte tratou de assuntos mais específicos ao problema da autoadministração de medicamentos, como afeta suas atividades de vida diária e como tentam solucionar o problema. 
Quadro 1 - Questões para orientar conversas Informais.

\begin{tabular}{|l|l|}
\hline 1a parte: Indivíduo & 2a parte: Questões Específicas ao problema \\
\hline - quanto tempo faz que o Sr.(a) tem problema na visão & - o que mudou após a perda de visão \\
- como classifica a própria visão & - se administra seus remédios sozinho \\
- se sabe qual tipo de tratamento já realizou nos olhos & - como organiza seus remédios \\
- quantos tipos diferentes de remédio toma & -como diferencia um remédio do outro \\
- se tem algum problema de saúde & \\
\hline
\end{tabular}

Fonte: Elaborado pelo autor, com base na pesquisa realizada

\subsection{Local e Amostra}

As conversas informais foram realizadas na sala de espera dos ambulatórios de Retina e Mácula do Departamento de Oftalmologia da Universidade Federal de São Paulo-UNIFESP, localizado na Rua Botucatu, no 817, São Paulo/SP. Foram entrevistados 25 pacientes com 60 anos ou mais, escolhidos ao acaso, por um período de 2 meses.

\subsection{Técnicas para compreender o problema}

Posterior às conversas foi utilizado a técnica do Stakeholder mapping para auxiliar na compreensão do problema e subproblemas envolvidos na pesquisa. Esta técnica consiste em um diagrama que representa o mapeamento das pessoas envolvidas no processo de design e contribui para enxergar o todo sem que o foco se desvie para a tecnologia mas sim continue nas pessoas (Bryson, 2007).

\section{RESULTADOS}

Este tipo de abordagem foi importante para o entendimento da realidade destes idosos. O dia a dia enfrentado por estes pacientes é complexo. Este tipo de abordagem favoreceu para que relatos sinceros aflorassem na conversa, e se aproximassem da realidade de como lidam com o problema e almejam possíveis soluções. Para estabelecer uma organização mais clara do material de pesquisa e descobertas, o material resultante das questões foi dividido em duas partes considerando os assuntos listados no Quadro 1.

\subsection{Parte I: Indivíduo}

Fatos que apontam para um dia a dia duro e difícil. Assuntos abordados serviram para compreender como o paciente lida com sua saúde, a atenção e julgamento sobre os procedimentos médicos que são realizados para melhorar esta condição e a percepção de tempo e tipo de doença.

A noção de tempo de perda visual em todos os pacientes é bastante acurada. Nenhum paciente avaliou sua visão como boa, e a grande maioria avaliou como ruim. Nesta fase da conversa foi possível constatar que alguns pacientes só descobrem que tem diabetes após o diagnóstico da perda da visão por RD. Muitos não sabem se um dia irão melhorar, mas tem noção dos tratamentos realizados para atenuar o problema visual. Todos também sabem listar quantidade e da função dos remédios que tomam, além da consciência de todos os outros problemas de saúde que possuem. A quantidade de remédios por paciente varia de 3 a 9 . Os problemas de saúde mais comuns foram: gastrite, pressão alta, diabetes e colesterol alto. 


\subsection{Parte II: Questões Específicas}

É perceptível o nível de descontentamento de todos os pacientes em relação às suas atividades de vida diária. A dependência de terceiros é muito grande, tanto para auxiliar em atividades ordinárias como para organizar e algumas vezes tomar o medicamento. Todos sentem falta da autonomia e independência que antes possuíam, para realizar atividades de vida diária (Quadro 2). A maioria relatou que os medicamentos distribuídos pelo Sistema Único de Saúde - SUS não vem em caixas, e alguns pacientes relataram que os medicamentos que compram na farmácia possuem embalagem, o que auxilia na distinção e organização dos medicamentos.

Estes idosos, além de múltiplos medicamentos, também utilizam insulina injetável e colírios. Os horários dos medicamentos são diversos, e alguns relataram que não possuem auxilio de familiares ou dependem da ajuda de amigos e vizinhos para ajudar em tarefas do dia a dia, como tomar remédios.

Quadro 2 - 0 que mudou na vida dos pacientes após a perda (total/parcial) da visão.

\begin{tabular}{|l|}
\hline Relatos dos pacientes \\
\hline "Praticamente tudo. Estou desanimado e só reconheço as pessoas pela voz" \\
\hline "Estou muito triste. Não consigo mais escrever, ler jornal, e cozinhar" \\
\hline "Não consigo mais ler jornal, e não trabalho mais. Não faço mais nada. Não saio sozinha na rua" \\
\hline "Mudou tudo. Não consigo mais cozinhar, escolher feijão nem arroz" \\
\hline "Mudou tudo. Não leio e não dirijo mais. Não faço mais nada. Não consigo ver cor" \\
\hline "Costurar, descascar legumes, assistir TV e distinguir o dinheiro" \\
\hline "Só leio com lupa e não trabalho mais. Não consigo mais passear e andar de escada rolante" \\
\hline "Não consigo abrir lata, não leio mais e não consigo ver nem TV" \\
\hline "Não leio nem escrevo mais. Não consigo tocar nenhum instrumento musical" \\
\hline "Mudou tudo. Estou com depressão. Até perdi peso. Preciso de ajuda até para limpar a casa" \\
\hline "Não leio mais. Preciso usar bengala e não posso mais trabalhar" \\
\hline "Limitou tudo. Não trabalho mais. Não assisto mais TV, só escuto. Não leio mais jornal” \\
\hline "Preciso de ajuda para tudo, até para andar, tenho medo de cair. TV só de perto. Ler só com lupa." \\
\hline
\end{tabular}

Fonte: Elaborado pelo autor, com base na pesquisa realizada

A falta de "padrão das embalagens" dos medicamentos distribuídos pelo SUS foi uma queixa constante, pois transformou-se em um problema. Pois muitos idosos utilizam a ação combinada de recursos sensoriais para distinção dos medicamentos como textura do comprimido, formato da cartela e cor dos comprimidos (Tabela 1).

Tabela 1 - Modo de distinção dos remédios pelos pacientes

\begin{tabular}{l|l|l|l|l|l|l}
\hline & $\begin{array}{l}\text { Cor do } \\
\text { comprimido }\end{array}$ & $\begin{array}{l}\text { Tamanho do } \\
\text { comprimido }\end{array}$ & $\begin{array}{l}\text { Textura do } \\
\text { comprimido }\end{array}$ & $\begin{array}{l}\text { Lugar onde } \\
\text { guarda }\end{array}$ & $\begin{array}{l}\text { Cartela de } \\
\text { comprimido }\end{array}$ & $\begin{array}{l}\text { Usa } \\
\text { lupa }\end{array}$ \\
\hline No de pacientes & 20 & 20 & 5 & 8 & 7 & 5 \\
\hline
\end{tabular}

Fonte: Elaborado pelo autor, com base na pesquisa realizada

Todos tentam criar seus próprios métodos de organização (Quadro 3) para de alguma forma facilitar a administração e tentar reduzir a dependência de terceiros. Muitos destes métodos necessitam do auxilio de familiares ou amigos para organizar. 
Quadro 3 - Métodos de organização dos medicamentos relatados pelos pacientes.

Diferentes métodos de organização dos medicamentos

anda com eles no bolso e em casa organiza em copos

separa remédios do dia e noite em copos com cores diferentes

separa em caixa com espaços para cada dia

copinhos e lugares diferentes da casa para os remédios do dia e da noite

tem um potinho com o nome do remédio (usa lupa)

retira da cartela e separa em potinhos com diferentes cores e estampas

insulina na geladeira e remédio em cima dela

organiza em envelopes e caixas sempre no mesmo lugar

Fonte: Elaborado pelo autor, com base na pesquisa realizada

\subsection{Stakeholder mapping}

Este paciente tem o apoio de familiares e amigos para realizar tarefas diárias que vão desde cozinhar, até pegar uma condução para ir até a consulta médica. 0 médico além de tratar suas doenças, explica e passa informações importantes sobre o processo de cura. $O$ posto de saúde tem a função tanto de distribuir medicamentos como de instruir o paciente e familiares sobre os diferentes remédios e seus aspectos técnicos. O designer utilizando uma abordagem centrada no humano se envolve em compreender esta rotina para criar e desenvolver soluções que visem melhorar o dia a dia destes idosos. O governo busca, através de programas, ensinar a importância do tratamento e fazer com que medicamentos sejam mais acessíveis. $O$ meio acadêmico se esforça para pesquisar, relacionar partes interessadas em estudos mais profundos sobre o problema levantado. Este idoso se conecta com todas estas partes envolvidas, tendo ou não o apoio, serviço ou atendimento.

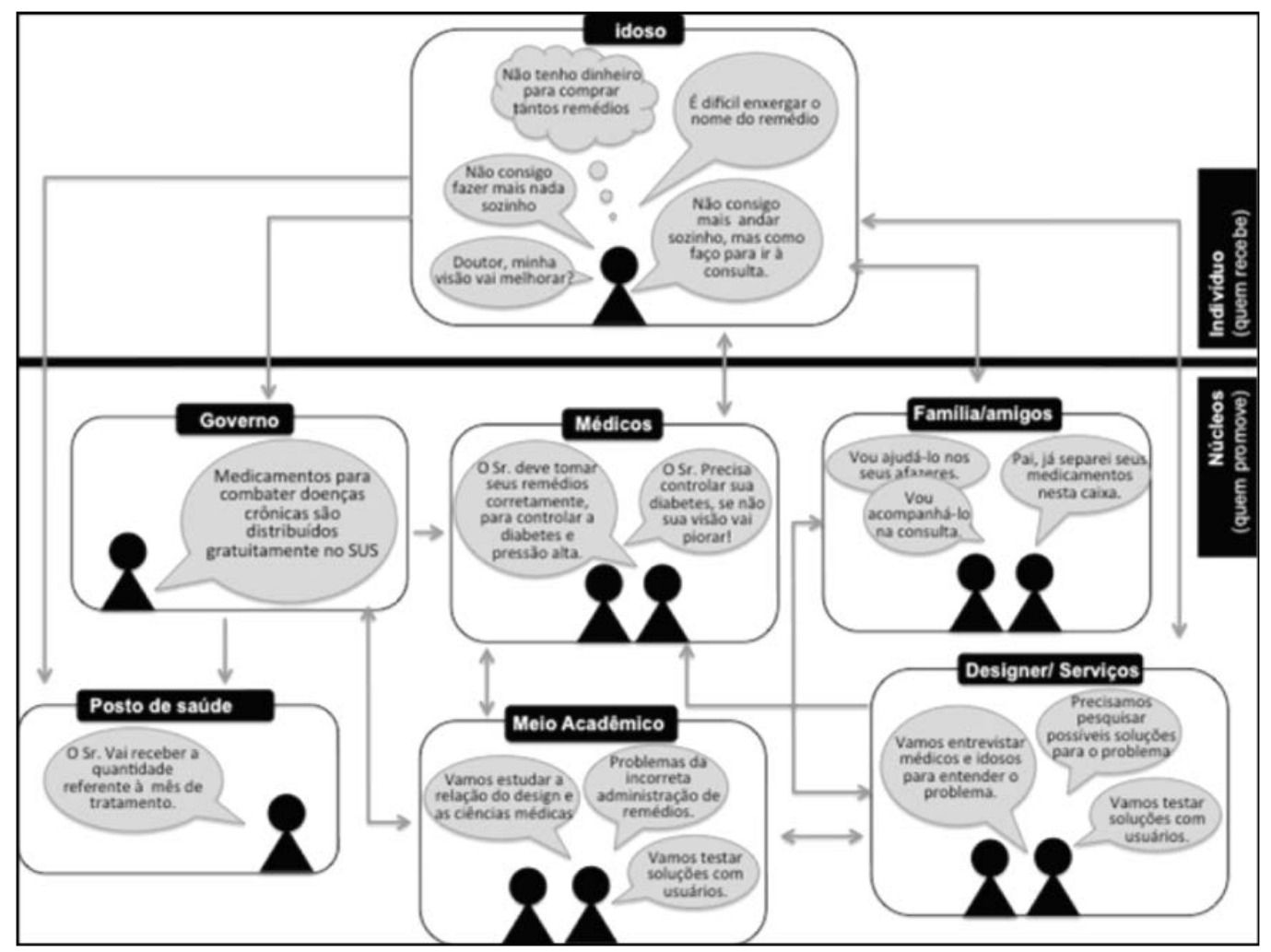

Figura 2 - Stakeholder mapping ou Mapeamento das partes envolvidas. Adaptado (Bryson, 2007) 


\subsection{Pré-requisitos para soluções futuras}

Todo material resultante das conversas informais com os pacientes, acompanhantes e médicos, e técnicas para definição e compreensão do problema, foi avaliado e analisado. Sequencialmente foram listados pré-requisitos para futuras soluções que visem a melhora da rotina de idosos com deficiência visual:

$\&$ esteticamente amigável, interativo e agradável ao toque

\& simples, intuitivo, de fácil uso e portátil

\& lúdico, gerar emoções e afeto

$\&$ inovador e explorar os sentidos remanescentes para comunicar por meio de outros canais perceptivos

\& ser autoexplicativo e assegurar que o paciente participe do seu próprio tratamento

\& mudar a relação do paciente com sua doença e tratamento

\& promover independência

\section{DISCUSSÃO}

A mudança da realidade demográfica mundial é um fato que aponta para essencial transformação do olhar da sociedade para o público da terceira idade. A reconstrução e criação de novas soluções e ações em diferentes frentes para suprir e incluir este público é urgente.

Avaliar o meio ambiente em seus diversos planos é primordial para compreender o ser humano em sua plenitude. E para isto a eficiência de abordagens transdisciplinares e centradas no usuário se mostra efetiva e completa, pois lida com o problema no plano do indivíduo e não da solução. O pesquisador interage com os seres humanos e suas reais dificuldades, e não cria "personas" fictícias para assim projetar. Assim, a possibilidade de agregar os U/E no processo de pesquisa foi essencial para aprofundar a compreensão de suas realidades e melhor desenhar 0 problema.

Patton (2001) afirma que dados recolhidos a partir de entrevistas de conversas informais são diferentes para cada pessoa entrevistada. Nesta fase portanto, por mais que muitos dados diferissem de paciente para paciente, uma constante foi possível detectar, o problema da autodministração dos medicamentos. Assim, as conversas com o público produziram dados que depois de confrontados com bibliografia e conversas com especialistas permitiu ao pesquisador imergir e compreender o universo deste idoso. A lista de questões auxiliou na condução das conversas informais, e foi um facilitador para compreender o problema. As perguntas foram montadas para não serem invasivas, buscando deixar o paciente confortável para falar e não se sentir "interrogado", respeitando assim suas fragilidades.

Alguns pacientes relataram que descobriram a diabetes somente quando começaram a ter problemas para enxergar devido à retinopatia diabética. Fato este que denota um grave problema de saúde pública, com pacientes mal informados sobre este mal crônico e seus efeitos nocivos ao corpo humano. Esta ignorância em conjunto com a uma notícia "repentina" e previsões negativas dos médicos sobre a deficiência de visão levam a um certo nível de prostração dos pacientes quando relatavam suas condições. Mesmo que ainda possuam certo auxílio de familiares ou amigos, ficou 
claro a falta de suporte psicológico dos pacientes, não possuindo resiliência necessária para enfrentar o problema e encarar a sua nova realidade de deficiente visual.

A habilidade dos pacientes para distinguir os medicamentos pela cor, tamanho e formato dos comprimidos e cartelas é impressionante. Ato que é prejudicado devido à falta de padrão dos comprimidos distribuídos pelo Sistema Único de Saúde - SUS. Muitos medicamentos distribuídos pelo sistema não vêm em caixas, mas somente em cartelas, e muitas vezes o mesmo medicamento apresenta características físicas distintas. Fato que aponta para um problema sistêmico de difícil controle para estes pacientes. A constância no aspecto físico dos medicamentos é uma ferramenta simples e intuitiva, que quase todos utilizam, mas quando inconstante, torna-se um fator de confusão que facilita a incorreta administração de medicamentos.

As soluções criadas pelos pacientes para organizar seus medicamentos demonstram a criatividade para inventar comunicações alternativas que não dependem apenas de uma informação escrita.

O stakeholder mapping auxiliou na compreensão mais efetiva dos envolvidos no problema, para possíveis abordagens e investigações. Foi possível delinear como algumas partes envolvidas, como médicos e posto de saúde, poderiam realizar ações mais abrangentes em busca de prevenção e educação destes pacientes e seus problemas de saúde. Assim como mais e maiores pesquisas transdisciplinares para abordar o tema e ampliar o olhar da academia para o tema.

$O$ design centrado permite que as conversas informais identifiquem questões não alcançadas por simples entrevistas. A situação real vivenciada por indivíduos reais, deixa para trás a construção de 'personas', pois expõe detalhes e insights sobre o problema que enriquecem a pesquisa de forma ampla (Fletcher, 2016)). Este contato direto com os pacientes, propiciou a avaliação do problema para definir premissas para futuras soluções. Assim, delinear pré-requisitos ao final dos resultados foi uma consequência natural desta metodologia, que se baseia nas demandas genuínas do indivíduo, e trabalha "com" e não "para" o sujeito da pesquisa.

\section{CONCLUSÃO}

A relação dos usuários com o problema foi avaliada e apresenta resultados expressivos, com dados que apontam para a necessidade de soluções que promovam a educação do paciente para sua doença e ao auxílio na distinção e administração medicamentos. Promovendo assim, a independência desta população e minimizando erros de autoadministração e suas consequências. Métodos de pesquisa qualitativa como conversas informais foram mais efetivos para a compreensão detalhada do pesquisador sobre as realidades enfrentadas pelos pacientes dos ambulatórios. Não tratar os U/E como "sujeitos de uma pesquisa" durante o estudo enriqueceu o processo de reconhecimento do problema, obtendo relatos mais francos de suas fraquezas e permitindo ao pesquisador traçar os pré-requisitos com mais clareza.

Os resultados sugerem serem necessárias mais pesquisas centradas no humano para compreender esta realidade, e indicam a importância de mais ações em diferentes frentes de pesquisa para lidar com este problema. Aprimorar a pesquisa transdisciplinar para criar e desenvolver soluções na área da saúde seria um primeiro grande começo. 


\section{REFERÊNCIAS}

BELON, A. P. E. A. Diabetes em idosos: perfil sócio-demográfico e uso de serviços de saúde. XVI Encontro Nacional de Estudos Populacionais, ABEP. Caxambu- MG: p 1-10p. 2008.

BONSIEPE, Gui. Design, cultura e sociedade. São Paulo: Blucher, 2011.

BRYSON, J. M. What to do when stakeholders matter. Stakeholders identification and analysis techniques UK: Francis \& Taylor Comp: 23-53 p. 2007.

CDC, Centers for Disease Control and Prevention. Blindness caused by diabetes-Massachusetts, 1987-1994. MMWR Morb Mortal Wkly Rep, v. 45, n. 43, p. 937-41, Nov 1996. Disponível em: <http://www.ncbi.nlm.nih.gov/pubmed/8927019>.

CHAVES, I. G. Estudo, análise e proposta de parâmetros e requisitos a serem considerados na metodologia de projeto do produto óculos para o público infantil 2014. (Mestrado). Design e Arquitetura, Faculdade de Arquitetura e Urbanismo (FAU) da Universidade de São Paulo (USP), São Paulo.

CLARKSON, P. J. et al. Inclusive Design: Design for the Whole Population. London: Springer, 2003.

EIKHAUG, O. et al. Innovating with people: The business of Inclusive Design. Oslo: Norsk Designrad, Norwegian Design Council, 2010.

FLETCHER, V. Consequential Design. National Endowment for the Arts. Washington, DC: Arts Works, 2014a. Disponível em:<https://www.arts.gov/art-works/2014/consequential-design>. Acessado em dezembro de 2014.

. Demographics, Climate + Disaster: Universal Design as a Framework for 21st Century Recovery Japan The 5th International Conference for Universal Design in Fukushima \& Tokyo. Keynote Oral Presentation. 2014b.

. inclusive/Universal Design, People at the Center of the Design Process. In: KANAANI, M. e KOPEC, D. (Ed.). The Rutledge companion for architecture design and practice: established and emerging trends: Routledge, 2016. p. 252-267.

FLETCHER, V.; CROLIUS, W. A.; HARADA, F.J.B. Improving Senior Ridership on Public Transit: An Approach to working with User/Experts to Review the Public Transportation for the City of Cambridge/MA with Emphasis on Universal Design. 2015 Universal Design Symposium. NC State College of Design, 2015.

FROYEN, H. Universal Design, a methodological approach, a pathway to human-friendly and elegant architecture. Hasselt, Belgium: Institute for Human Centered Design - IHCD, 2012.

GIACOMIN, J. What is Human Centered Design? 10 Congresso Brasileiro de Pesquisa e Desenvolvimento em Design. São Luís, MA, Brazil 2012.

GLOBAL AGEWATCH INDEX. Executive Summary 2015. This work is licensed under a Creative Commons Attribution-Non Commercial 4.0 International License. Disponível em: $<$ https://creativecommons.org/licenses/by-nc/4.0 . Acessado em março de 2016.

IBGE, Instituto Brasileiro de Geografia e Estatística. Brasil, expectativa de visa ao nascer 2002030, 2008.

ILC, I. L. C. B. ACTIVE AGEING:A Policy Framework in Responseto the Longevity Revolution. 1st edition. Rio de Janeiro, RJ, Brazil: ILC - Brasil, 2015. 
JANNUZZI, F. F. Qualidade de vida relacionada a função visual e adesão medicamentosa em idosos com retinopatia diabetica. 2009. (Dissertação mestrado). Programa de Pós-Graduação em Enfermagem, Universidade Estadual de Campinas . Faculdade de Ciências Médicas, Campinas.

KALACHE, A. The Longevity Revolution. Creating a society for all ages. Adelaide: Government of South Australia: Ed. Adelaide Thinker in Residence, 2013.

LEONARD, D.; RAYPORT, J. F. Spark Innovation Through Empathic Design. Harvard Business Review: p. $102-113,1997$.

LOYOLA FILHO, A. I. D.; UCHOA, E.; LIMA-COSTA, M. F. Estudo epidemiológico de base populacional sobre uso de medicamentos entre idosos na Região Metropolitana de Belo Horizonte, Minas Gerais, Brasil. . Cad. Saúde Pública. Rio de Janeiro. v. 22: p. 2657-2667, 2006.

MCCANN, R. M. et al. Help needed in medication self-management for people with visual impairment: case-control study. Br J Gen Pract, v. 62, n. 601, p. e530-7, Aug 2012. Disponível em: < http://www.ncbi.nlm.nih.gov/pubmed/22867676 >. Acessado em janeiro de 2016.

MENDONÇA, R. H. F. D. et al. Qualidade de vida em pacientes com retinopatia diabética proliferativa. Revista Brasileira de Oftalmologia, v. 67, p. 177-183, 2008. Disponível em: < http://www.scielo.br/scielo.php?script=sci_arttext\&pid=S0034-72802008000400004\&nrm=iso >. Acessado em julho de 2014.

NORMAN, D. A. The Design Everyday Things. New York: Basic Books, 2002. Acessado em maio de 2014.

PATTON, M. Q. Qualitative Research \& Evaluation Methods. 3rd ed. United States of America: Sage Publications, Inc., 2001.

POSTMA, C. et al. Challenges of Doing Empathic Design: Experiences from Industry. International Journal of Design [Online] 6:1, p59-70, 2012.

SAMPAIO, M. W.; HADDAD, M. A. O. Baixa Visão: Manual para o Oftalmologista Rio de Janeiro: Cultura Médica: Guanabara Koogan, 2009.

SURI, J. F. Informed and inspired by other people's experience. In: KOSKINEN, I. B. e K.MATTELMÄKI, T. (Ed.). Empathic design: User Experience in Product Design. Helsinki, Finland: IT Press, 2003. cap. Informed and inspired by other people's experience, p.pp. 51-58.

TURATO, E. R. Tratado da Metodologia da pesquisa clínico-qualitativa: construção teóricoepistemológica, discussão comparada e aplicação nas áreas da saúde e humanas. . 3 ed. Petrópolis, RJ: Vozes, 2008.

ZAITUNE, M. P. D. A. et al. Hipertensão arterial em idosos: prevalência, fatores associados e práticas de controle no Município de Campinas, São Paulo, Brasil. Cadernos de Saúde Pública, v. 22, p. 285-294, 2006. Disponível em:<

http://www.scielo.br/scielo.php?script=sci_arttext\&pid=S0102-311X2006000200006\&nrm=iso $>$. Acessado em novembro de 2014. 